\title{
Recent Developments in Automated Spectral Image Analysis
}

\author{
M.R. Keenan* and P.G. Kotula* \\ *Sandia National Laboratories, PO Box 5800, Albuquerque, NM 87185-0886
}

Several full-spectrum imaging techniques have been introduced, in recent years, that promise to provide rapid and comprehensive chemical characterization of complex samples. New imaging systems allow the collection of a complete spectrum at each point in a 1-, 2- or 3-D spatial array. One remaining obstacle to adopting these techniques for routine use is the daunting task of reducing the vast quantities of raw spectral data to meaningful chemical information. Factor analysis techniques have proven effective for extracting the chemical information from high dimensional data sets into a limited number of components that describe the spectral characteristics and spatial distributions of the chemical species comprising the sample. These methods suffer, however, from a "rotational ambiguity", that is, there are an infinite number of factor models that will describe the data equally well. The key to deriving easily interpretable components is to constrain the factor solutions to conform to physical reality. The method described by Kotula, for instance, employs an Alternating Least Squares-based Multivariate Curve Resolution (MCR-ALS) technique that imposes nonnegativity on component spectra and abundances [1]. While this approach has proven remarkably successful, there are many cases in which such constraints are not effective and alternative procedures are required.

A simple case in point is illustrated in Fig. 1. The sample consists of a polysilicon structure built up on a silicon nitride substrate. The sample was sputtered with argon ions to remove a surface oxide and was imaged by X-ray Photoelectron Spectroscopy (XPS). The data set comprises an 801-channel spectrum at each point in the 256 x 256-pixel image, and the average of the 65536 total spectra is also shown in Fig. 1. Eigenanalysis of the spectral data crossproduct matrix suggests that 5 components are required to fully describe the data. The results of a standard non-negativity-constrained MCR-ALS analysis are shown in Fig. 2a. These results are less than satisfactory. The estimated backgrounds generally have non-realistic shapes, and several spectroscopic features, while strictly non-negative, are negative with respect to the background. Additionally, it appears that two components are required to describe each of the polysilicon and silicon nitride phases. A careful examination of the derived components shows that the spectra exhibit slight, spatially correlated energy shifts, and the pure-component spectra span the range of that shift. The results of an alternative data analysis approach are shown in Fig. 2b. In this case, MCR-ALS was performed with the addition of "spatial simplicity" constraints, and the energy shift was accommodated by equality constraining two spectral components to equal the derivatives of their respective pure-component spectra. These latter equality constraints serve to separate the chemical information from the instrumental artifact. The "spatial simplicity" constraints, on the other hand, exploit the fact that many samples are simple in the sense that only one or a few components will be present in any given pixel. The three chemically relevant pure components in Fig. $2 \mathrm{~b}$ show improved contrast in their abundance maps and more realistic spectral shapes as compared to the standard MCR-ALS estimates. The pure components now clearly represent the polysilicon and silicon nitride phases, as well as, an oxide phase present along edges that were shadowed during sputtering [2-3].

References:

[1] P.G. Kotula, M.R. Keenan, J.R. Michael, Microsc. Microanal. 9 (2003) 1-19.

[2] The authors would like to thank Tony Ohlhausen for providing the XPS data.

[3] Sandia is a multiprogram laboratory operated by Sandia Corporation, a Lockheed Martin Company, for the United States Department of Energy's National Nuclear Security Administration under Contract DE-AC04-94AL85000. 

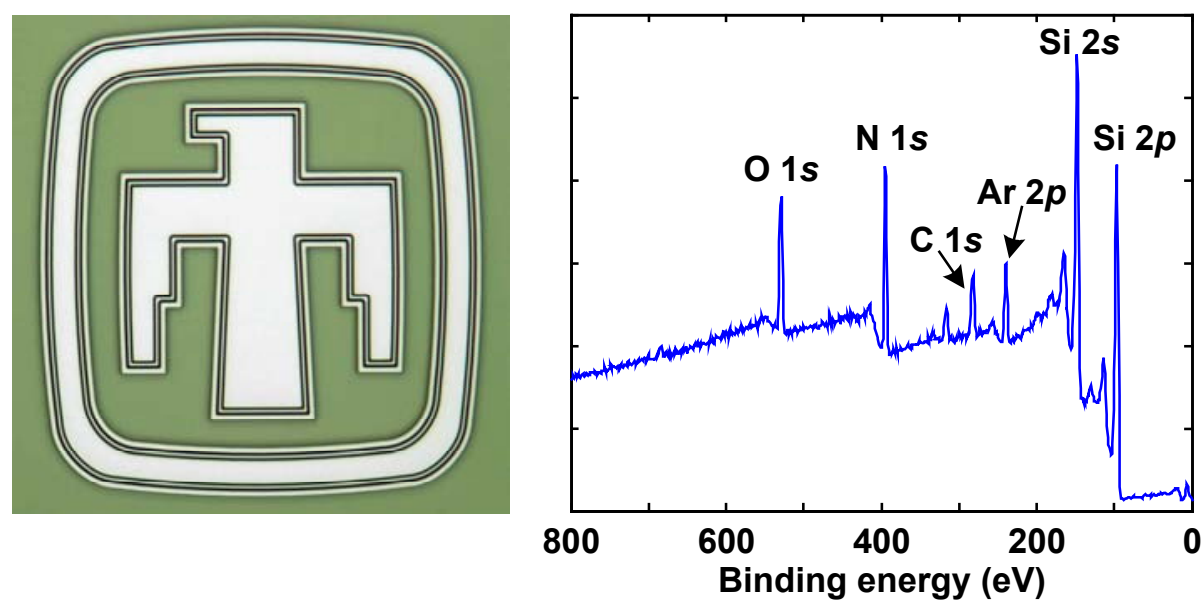

FIG. 1. Illustration of a polysilicon (light) on silicon nitride (dark) sample, together with its mean XPS spectrum.

(a)
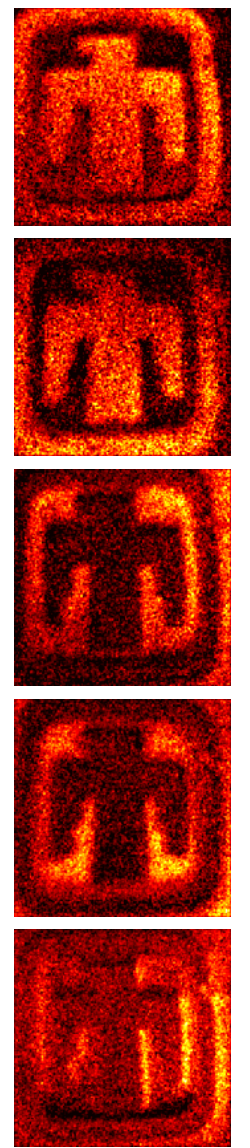
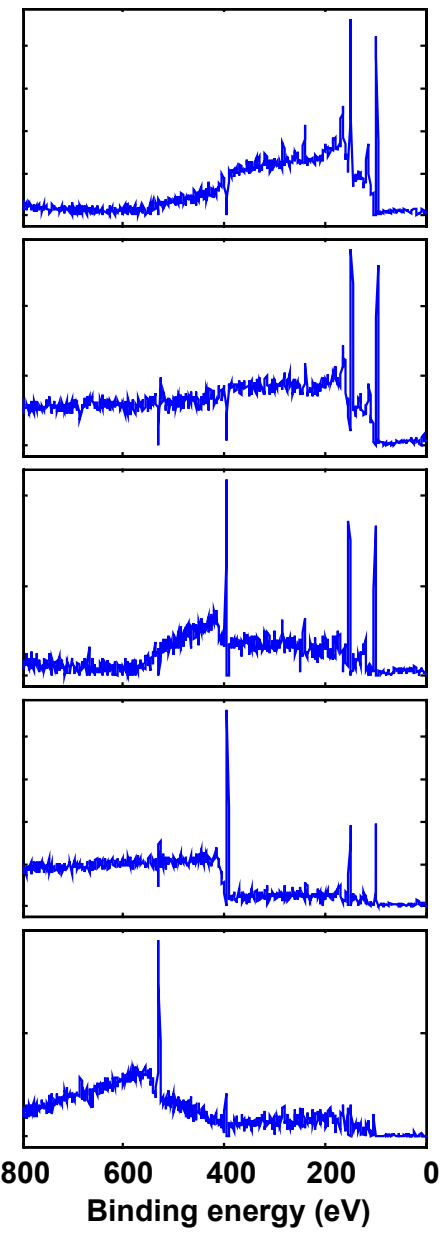

(b)
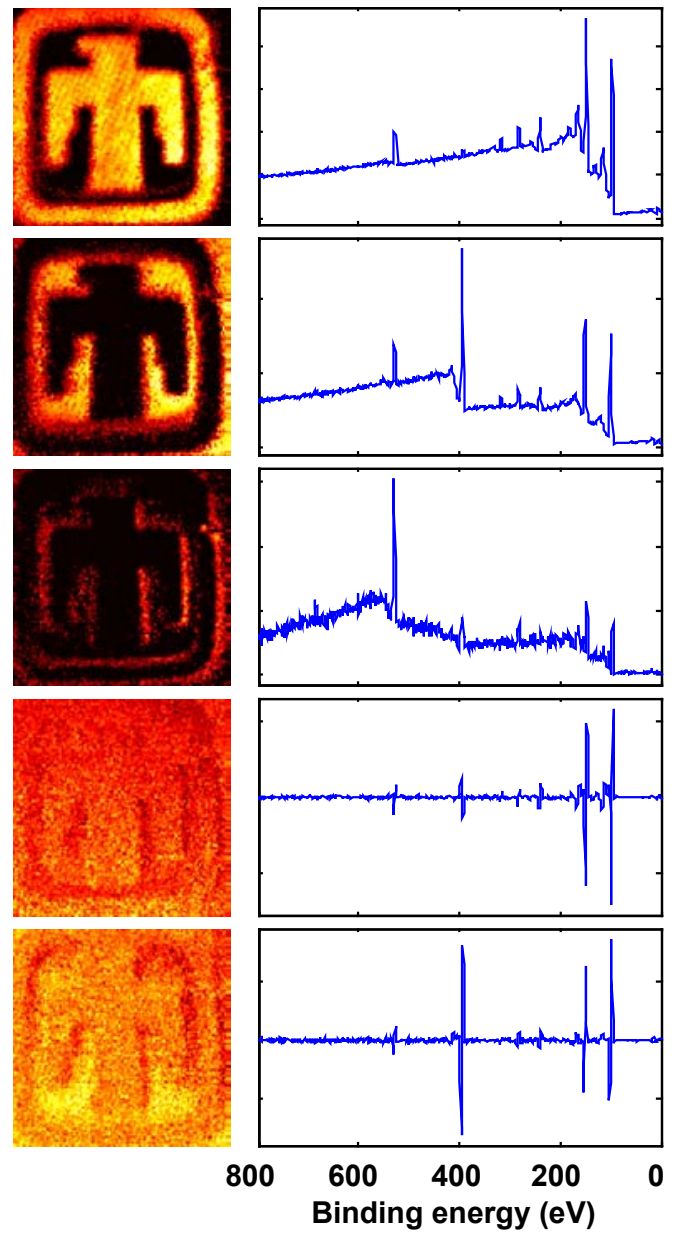

FIG. 2. MCR-ALS results for the XPS spectral image using (a) non-negativity constraints alone and (b) non-negativity, spatial simplicity and spectral equality constraints. 